PROCEEDINGS OF THE

AMERICAN MATHEMATICAL SOCIETY

Volume 127, Number 7, Pages 2053-2057

S 0002-9939(99)04752-8

Article electronically published on February 17, 1999

\title{
CLASSIFICATION OF ACTIONS OF DISCRETE AMENABLE GROUPS ON STRONGLY AMENABLE SUBFACTORS OF TYPE III $_{\lambda}$
}

\author{
TOSHIHIKO MASUDA
}

(Communicated by Palle E. T. Jorgensen)

\begin{abstract}
Using the continuous decomposition, we classify strongly free actions of discrete amenable groups on strongly amenable subfactors of type $\mathrm{III}_{\lambda}$, $0<\lambda<1$. Winsløw's fundamental homomorphism is a complete invariant. This removes the extra assumptions in the classification theorems of Loi and Winsl $\varnothing \mathrm{w}$ and gives a complete classification up to cocycle conjugacy.
\end{abstract}

\section{INTRODUCTION}

In the theory of operator algebras, the study of automorphisms is one of the most important topics. Especially since Connes's work [3], much progress has been made on the classification of the actions of discrete amenable groups on injective factors.

In the subfactor theory, various studies of automorphisms have been done. In [13], Popa has introduced the notion of proper outerness of automorphisms and proved that the properly outer actions of discrete amenable groups on strongly amenable subfactors of type $\mathrm{II}_{1}$ are classified by the Loi invariant (see [9]). (In [1], Choda and Kosaki have introduced the same property independently and they call it strong outerness.)

In the case of subfactors of type $\mathrm{III}_{\lambda}(0<\lambda<1)$, partial results on classification of group actions have been obtained by Winsløw and Loi ([10], [17], [18]). In [17] and [18], Winsløw has introduced the strong freeness and the fundamental homomorphism for actions. He has classified the strongly free actions of discrete amenable groups on subfactors of type $\mathrm{III}_{\lambda}$ for groups having the character lifting property. His fundamental homomorphism is a complete invariant. In [10], Loi gave a classification theorem when $G$ is finite. Their idea of the proof is that they reduce the classification problem to the type $\mathrm{II}_{\infty}$ case using the discrete decomposition and apply Popa's classification result. The most difficult points of their proofs are to reduce the problem to the type $\mathrm{II}_{\infty}$ case. Because of this difficulty, they made extra assumptions such as the character lifting property or finiteness for groups. But it seems difficult to generalize their method to the arbitrary discrete amenable group case.

Our idea of a proof is using the continuous decomposition instead of the discrete decomposition based on the method in [14] and [15]. But in this case, we treat

Received by the editors March 3, 1997 and, in revised form, October 3, 1997.

1991 Mathematics Subject Classification. Primary 46L37. 
only factors of type $\mathrm{III}_{\lambda}, 0<\lambda<1$, with the trivial characteristic invariants, so the proof is less complicated than those in [14] and [15]. By using the continuous decomposition, we can more easily reduce the classification problem to the type $\mathrm{II}_{\infty}$ case than using the discrete decomposition and this method is valid for arbitrary discrete amenable groups.

After the submission of the first version of this paper, we have obtained a classification result also for group actions on subfactors of type $\mathrm{III}_{0}$ in [11] in a more complicated method. But the simple argument in this paper is a key to a generalization in [11], and the author hopes that the short proof in this paper is of independent interest.

\section{Preliminaries}

In this section, we recall several results about group actions on subfactors, and fix notations. The facts stated in this section are found in [1], [9], [10], [13], [17], [18], [19].

Let $N \subset M$ be an inclusion of factors with finite index and $N \subset M \subset M_{1} \subset$ $M_{2} \subset \cdots$ the Jones tower. (Throughout this paper, we always assume that conditional expectations are minimal in the sense of [8] and inclusions of factors of type II are extremal.) For $\alpha \in \operatorname{Aut}(M, N)$, we extend $\alpha$ to $M_{k}$ such that $\alpha\left(e_{k}\right)=e_{k}$ inductively, where $e_{k}$ denotes the Jones projection for $M_{k-1} \subset M_{k}$.

First we recall the Loi invariant and the strong outerness of group actions.

Definition 2.1 ([9, Section 5]). With above notations, Put

$$
\Phi(\alpha):=\left\{\left.\alpha\right|_{M^{\prime} \cap M_{k}}\right\}_{k} .
$$

We call $\Phi$ the Loi invariant for $\alpha$.

Definition 2.2 ([1, Definition 1], [13, Definition 1.5.1]). An automorphism $\alpha \in$ $\operatorname{Aut}(M, N)$ is said to be properly outer or strongly outer if we have no non-zero $a \in \bigcup_{k} M_{k}$ satisfying $\alpha(x) a=a x$ for all $x \in M$. The action $\alpha$ of $G$ on $N \subset M$ is said to be strongly outer if $\alpha_{g}$ is strongly outer except for $g=e$.

The most important result on classification of actions of groups on subfactors has been obtained by Popa.

Theorem 2.3 ([13, Theorem 3.1]). Let $N \subset M$ be a strongly amenable inclusion of factors of type $I I_{1}$ and $G$ a countable discrete amenable group.

If $\alpha$ and $\beta$ are strongly outer actions of $G$ on $N \subset M$, then $\alpha$ and $\beta$ are cocycle conjugate if and only if $\Phi(\alpha)=\Phi(\beta)$.

For type $\mathrm{II}_{\infty}$ inclusions, we have the following result due to Popa and Winsløw.

Theorem 2.4 ([13, Theorem 2.1], [17, Theorem 4.3]). Let $N \subset M$ a strongly amenable inclusion of factors of type $I I_{\infty}$. If $\alpha$ and $\beta$ are actions of countable discrete amenable group $G$ on $N \subset M$, then $\alpha$ and $\beta$ are cocycle conjugate if and only if $\Phi(\alpha)=\Phi(\beta)$ and $\bmod (\alpha)=\bmod (\beta)$.

Let $N \subset M$ be an arbitrary inclusion of factors with the common flow of weights. Fix a normal state of $N$ and take a crossed product of $N \subset M$ by the modular automorphism. Put $\tilde{N} \subset \tilde{M}:=N \rtimes_{\sigma^{\phi}} \mathbf{R} \subset M \rtimes_{\sigma^{\phi \circ E}} \mathbf{R}$, where $E$ is the minimal conditional expectation from $M$ onto $N$. Let $\tilde{\alpha}$ be the canonical extension of $\alpha$ to $\tilde{N} \subset \tilde{M}$ ([5], [7]), i.e.

$$
\tilde{\alpha}(x):=\alpha(x), \quad x \in M,
$$




$$
\tilde{\alpha}(\lambda(t)):=\left(D \phi \circ \alpha^{-1}: D \phi\right)_{t} \lambda(t)
$$

where $\lambda(t)$ is the usual implementing unitary. The notions of strong freeness for automorphisms and the fundamental homomorphism are introduced by Winsløw in [17], [18].

Definition 2.5 ([17, Definition 3.2], [18, Definition 4.2]). An automorphism $\alpha \in$ $\operatorname{Aut}(M, N)$ is said to be strongly free if we have no non-zero $a \in \bigcup_{k} \tilde{M}_{k}$ satisfying $\tilde{\alpha}(x) a=a x$ for all $x \in \tilde{M}$. For an action $\alpha$ of $\mathrm{G}$ on $N \subset M$ is said to be strongly free if $\alpha_{g}$ is strongly free except for $g=e$.

According to [17] and [18], we set

$$
\Upsilon(\alpha):=\left\{\left.\tilde{\alpha}\right|_{\tilde{M}^{\prime} \cap \tilde{M}_{k}}\right\}_{k}
$$

and we call this the fundamental homomorphism.

\section{Classification of aCtions}

Throughout this section, we assume that inclusions of factors of type $\mathrm{III}_{\lambda}$ are strongly amenable in the sense of Popa. (See [12] and [13].)

The following theorem is the main result of this paper.

Theorem 3.1. Let $N \subset M$ be a strongly amenable inclusion of factors of type $I I I_{\lambda}, 0<\lambda<1$, with the common flow of weights. Let $G$ be a countable discrete amenable group, and $\alpha$ and $\beta$ strongly free actions of $G$ on $N \subset M$. Then $\alpha$ and $\beta$ are cocycle conjugate if and only if $\Upsilon(\alpha)=\Upsilon(\beta)$.

Our idea of proof is that we lift actions to inclusions of type $\mathrm{II}_{\infty}$ von Neumann algebras using continuous decomposition and apply Popa's result.

The "only if" part is obvious, so we only prove the "if" part. Let $\left(X, F_{t}\right)$ be the flow of weights of $M$. Since $M$ is of type $\operatorname{III}_{\lambda},\left(X, F_{t}\right)$ is of the form $([0,-\log \lambda)$, translation). And we have an isomorphism

$$
\left(\tilde{N} \subset \tilde{M} \subset \tilde{M}_{1} \subset \cdots\right) \cong\left(L^{\infty}(X) \otimes Q \subset L^{\infty}(X) \otimes P \subset L^{\infty}(X) \otimes P_{1} \subset \cdots\right),
$$

where $Q \subset P \subset P_{1} \subset \cdots$ is a tower of factors of type $\mathrm{II}_{\infty}$ and $Q \subset P$ is strongly amenable by assumption.

Let $\theta_{t}$ be the usual trace scaling action of $\mathbf{R}$ on $\tilde{N} \subset \tilde{M}$. Since $\tilde{\alpha}_{g}, g \in G$, commutes with $\theta_{t}$, we can consider an action of $G \times \mathbf{R}$ by setting $(g, t) \rightarrow \tilde{\alpha}_{g} \theta_{t}$. If no confusion arises, we also denote this action of $G \times \mathbf{R}$ by $\tilde{\alpha}$. If we prove that two actions of $G \times \mathbf{R}, \tilde{\alpha}$ and $\tilde{\beta}$ are cocycle conjugate, the proof of [15, Proposition 1.1] also works in this case and we can deduce that the canonical extensions of $\tilde{\alpha}$ and $\tilde{\beta}$ on $N \rtimes_{\sigma^{\phi}} \mathbf{R} \rtimes_{\theta} \mathbf{R} \subset M \rtimes_{\sigma^{\phi \circ E}} \mathbf{R} \rtimes_{\theta} \mathbf{R} \cong N \otimes B\left(L^{2}(\mathbf{R})\right) \subset M \otimes B\left(L^{2}(\mathbf{R})\right)$ are also cocycle conjugate and get the conclusion that $\alpha$ and $\beta$ are cocycle conjugate, since $N$ and $M$ are properly infinite.

So our purpose is the classification of actions of $G \times \mathbf{R}$ on $\tilde{N} \subset \tilde{M}$. Note that for $g \in G$, the equality $\operatorname{tr}_{\tilde{M}} \tilde{\alpha}_{g}=\operatorname{tr}_{\tilde{M}}$ holds.

Put $H:=G \times \mathbf{R}$ and we consider the action $\tilde{\alpha}$ of the groupoid $H \ltimes X$ on $Q \subset P$ by the equality

$$
\tilde{\alpha}_{g}(a):=\int_{X}^{\oplus} \tilde{\alpha}_{\left(g, g^{-1} x\right)}\left(a\left(g^{-1} x\right)\right) d x \quad \text { (see [15, Proposition 1.2]). }
$$

Put $x_{0}:=0 \in X$ and $H_{0}:=\left\{\left(g, x_{0}\right) \in H \ltimes X \mid g x_{0}=x_{0}\right\}$. Then $H_{0}$ is a discrete amenable group acting on $Q \subset P$. For $x \in X=[0,-\log \lambda)$, we define 
$h(x): X \rightarrow X$ by $h(x) y:=y+x$, where sum is taken modulo $-\log \lambda$. Especially $h(x) x_{0}=x$.

Here we have the following proposition.

Proposition 3.2. If the action of $G$ is strongly free, then the action of $H_{0}$ is strongly outer.

Proof. Assume that the action of $H_{0}$ is not strongly outer. Then there exists $g \in H \backslash\{e\}$ and a non-zero $a \in P_{k}$ for some $k$ such that for every $b \in Q$, we have $\tilde{\alpha}_{g}(b) a=a b$. Since $\tilde{\alpha}_{g}$ is not strongly outer and $\bmod \tilde{\alpha}_{g}=1$, we know that $g$ is in $G$.

Set

$$
\tilde{a}:=\int_{X}^{\oplus} \tilde{\alpha}_{\left(h(x), x_{0}\right)}(a) d x .
$$

Then an easy computation shows that the equality $\tilde{\alpha}_{g}(b) \tilde{a}=\tilde{a} b$ holds for every $b \in Q$ and this means that action $\alpha$ is not strongly free.

Proof of Theorem 3.1. Let $\alpha$ and $\beta$ be strongly free actions of $G$ on $N \subset M$ such that $\Upsilon(\alpha)=\Upsilon(\beta)$. Then we get two actions $\tilde{\alpha}$ and $\tilde{\beta}$ of the same groupoid $H \ltimes X$. So we get two actions $\tilde{\alpha}$ and $\tilde{\beta}$ of a discrete amenable group $H_{0}$.

By Proposition 3.2, both actions are strongly outer and by assumption both actions have the same Loi invariant and the same module. So there exists an automorphism $\theta \in \operatorname{Aut}(P, Q)$ and $u_{g} \in Z_{\tilde{\beta}}\left(H_{0}, U(Q)\right)$ such that

$$
\theta \tilde{\alpha}_{g} \theta^{-1}=\operatorname{Ad} u_{g} \tilde{\beta}_{g}, \quad g \in H_{0}
$$

Set

$$
\begin{gathered}
\theta_{x}:=\tilde{\beta}_{\left(h(x), x_{0}\right)} \theta \tilde{\alpha}_{\left(h(x), x_{0}\right)}^{-1}, \quad x \in X, \quad \text { and } \\
u_{(g, x)}:=\tilde{\beta}_{\left(h(g x)^{-1} g h(x), x_{0}\right)}\left(u_{h(g x)^{-1} g h(x)}\right), \quad(g, x) \in H \ltimes X .
\end{gathered}
$$

Then an easy computation shows that $u_{(g, x)} \in Z_{\tilde{\beta}}(H \ltimes X, U(Q))$ and the equality

$$
\theta_{g x} \tilde{\alpha}_{g, x} \theta_{x}^{-1}=\operatorname{Ad} u_{(g, x)} \tilde{\beta}_{(g, x)}
$$

holds.

Put $\tilde{\theta}:=\int_{X}^{\oplus} \theta_{x} d x$ and $\tilde{u}_{g}:=\int_{X}^{\oplus} u_{(g, x)} d x$. Then we get

$$
\tilde{\theta} \tilde{\alpha}_{g} \tilde{\theta}^{-1}=\operatorname{Ad} \tilde{u}_{g} \tilde{\beta}_{g}
$$

and we get the conclusion.

\section{ACKnowledGement}

The author is grateful to Prof. M. Izumi for proposing this problem to him and for helpful suggestions, and to Prof. Y. Kawahigashi for fruitful comments and constant encouragement. He also thanks the editor and the referee for kind comments on this paper. 


\section{REFERENCES}

1. Choda, M., and Kosaki, H., Strongly outer actions for an inclusion of factors, J. Func. Anal. 122, 315-332, (1994). MR 96b:46085

2. Connes, A., Une classification des facteurs de type III, Ann. Sci. Ec. Norm. Sup. 6, 133-252, (1973). MR 49:5865

3. Connes, A., Outer conjugacy classes of automorphism of factors, Ann. Sci. Ec. Norm. Sup. 8, 383-420, (1975). MR 52:15031

4. Connes, A., Classification of injective factors, Ann. Math. 104,73-115, (1976). MR 56:12908

5. Connes, A., and Takesaki, M., The flow of weights on factors of type III, Tohoku Math. J. 29, 473-575, (1977). MR 82a:46069a

6. Jones, V. F. R., Index for subfactors, Invent. Math. 72, 1-25, (1983). MR 84d:46097

7. Haagerup, U. and Størmer, E., Equivalences of normal states on von Neumann algebras and the flow of weights, Adv. Math. 83, 180-262, (1990). MR 92d:46150

8. Hiai, F., Minimizing indices of conditional expectations onto a subfactor, Publ. RIMS. 24, 673-678, (1990). MR 90a:46157

9. Loi, P. H., On automorphism of subfactors, J. Func. Anal. 141, 275-293, (1996). CMP 97:04

10. Loi, P. H., Periodic and strongly free automorphisms of finite groups on inclusions of $I I I_{\lambda}$ factors, Internat. J. Math. 8, 83-96. (1997) MR 87k:46072

11. Masuda, T., Classification of strongly free actions of discrete amenable groups on strongly amenable subfactors of type $I I I_{0}$, preprint (1997).

12. Popa, S., Classification of amenable subfactor of type II Acta. Math. 172, 163-255, (1994). MR 95f:46105

13. Popa, S., Classification of actions of discrete amenable groups on amenable subfactors of type II, preprint (1992).

14. Sutherland, C. E. and Takesaki, M., Action of discrete amenable groups and groupoids on von Neumann algebras, Publ. RIMS. 21, 1087-1120, (1985). MR 88h:46125

15. Sutherland, C. E. and Takesaki, M., Action of discrete amenable groups on injective factors of type $I I I_{\lambda}, \lambda \neq 1$, Pac. J. Math. 137, 405-444, (1989). MR 90k:46142

16. Takesaki, M., Duality for crossed products and the structure of von Neumann algebra of type III, Acta. Math. 131, 249-310, (1973). MR 55:11068

17. Winsløw, C., Strongly free actions on subfactors, Internat. J. Math. 4, 675-688, (1993). MR 94i: 46080

18. Winsløw, C., Approximately inner automorphism on inclusions of type $I I I_{\lambda}$ factors, Pac. J. Math. 166, 385-400, (1994). MR 95m: 46102

19. Winsløw, C., Automorphism on an inclusion of factors, Subfactors - Proceeding of the Taniguchi Symposium, Katata —, (ed, H. Araki, et al.), World Scientific, 139-152, (1994). MR 96c:46063

Department of Mathematical Sciences, University of Tokyo, Komaba, Tokyo, 153, JAPAN

E-mail address: masuda@ms.u-tokyo.ac.jp 